\section{Brentuximab vedotin therapy for cutaneous lesions in T-prolymphocytic leukemia: a case report}

\author{
Jordan Senchak, ${ }^{1}$ Peter Pickens ${ }^{2}$ \\ ${ }^{1}$ Drexel University College of Medicine, \\ Philadelphia; ${ }^{2}$ Department of \\ Hematology/Oncology, Abington \\ Memorial Hospital, Abington, PA, USA
}

\begin{abstract}
We present an 88-year-old male with simultaneous T-cell prolymphocytic leukemia and stable smoldering myeloma with excellent initial response to three months of alemtuzumab. The patient relapsed at twelve months with severe cutaneous disease. Biopsy of a representative plaque demonstrated CD30 positivity in rare malignant lymphocytes. The patient demonstrated no response to reintroduction with a full course of alemtuzumab. He was therefore treated with brentuximab vedotin, resulting in partial remission of skin involvement that persisted for three months.
\end{abstract}

\section{Introduction}

T-cell prolymphocytic (T-PL) is a rare, mature, and aggressive T-cell neoplasm involving multiple organ systems, including blood, bone marrow, spleen, and lymph nodes. T-PL comprises $2-5 \%$ of mature lymphocytic leukemia in adult patients. ${ }^{1}$ Older adults are most often affected, with a median age of $61 .^{2}$ Patients most often present with hepatosplenomegaly, lymphocytosis, and generalized lymphadenopathy. Cutaneous manifestations in T-PL are common, consisting of rash, papules, purpura, and ulcers. ${ }^{3}$ Presentation of the skin lesions can range from initial diagnosis up to two years post diagnosis. ${ }^{3}$ Alemtuzumab is the first line therapy for T-PL, as most cases are CD52 positive. Despite alemtuzumab efficacy in treatment of T-PL, median overall survival is only one year, with less than a $10 \%$ survival at 5 years, and a relapse rate of nearly $100 \%{ }^{4-7}$ Although our patient responded to alemtuzumab, he relapsed after roughly twelve months with severe cutaneous involvement, and was unresponsive to repeat therapy. Brentuximab vedotin (BV) is an antibody-conjugate consisting of a chimeric immunoglobulin specific for CD30, with cytotoxic auristatin E, and a protease covalent linker. ${ }^{5}$ This treatment provides efficient, targeted therapy against CD30 posi- tive tumor cells, and was effective in diminishing the cutaneous lesions seen in our patient after relapse.

\section{Case Report}

We report an 88-year-old man who presented with weight loss, paraneoplastic sweats, headaches and fatigue over a 3-4 month course in April 2014. Physical examination was remarkable for pallor and peripheral lymphadenopathy with an absence of splenomegaly and any cutaneous involvement. Bone marrow biopsy showed $45 \%$ cellularity with $30-35 \%$ T-cells that coexpressed CD4 and CD8 and were positive for CD52. Flow cytometry detected $40 \%$ abnormal $\mathrm{T}$ cells that expressed CD2, cytoplasmic but not surface CD3, CD4, CD5, CD7, and CD8. FISH analysis did not detect a TcR alpha/delta rearrangement, but did identify a $+14 q 32.33$ in an average of $28 \%$ of the nuclei, which raised the possibility of an upstream abnormality in the Tcell leukemia/lymphoma-1A (TCL1A)/TCL1B gene region, which is common in T-PL. Karyotyping showed a gain of chromosome 8 and deletion of 11q, classic features of T-PL. Immunostains showed 10\% kappa-restricted plasma cells, and flow cytometry detected a monoclonal kappa plasma cell population. These findings were consistent with a plasma cell neoplasm, and in the absence of myelomarelated organ or tissue damage represented smoldering myeloma. Myeloma FISH analysis did not detect abnormalities that are typically seen in malignant plasma cells. CBC showed a leukocytosis with an absolute lymphocyte count 6400 , white blood cell count of 20.0, $33.8 \%$ lymphs, 6\% monos, $60.2 \%$ PMNs, and low hemoglobin of 10.2. CT scan demonstrated extensive mesenteric lymphadenopathy and non-bulky axillary and mediastinal lymphadenopathy. Liver and spleen were normal in size. Skeletal survey did not demonstrate osteolysis. Treatment with $30 \mathrm{mg}$ alemtuzumab was started three times a week for 12 weeks. Following treatment with alemtuzum$\mathrm{ab}$, he demonstrated resolution of constitutional symptoms, resolution of lymphocytosis, and improvement of anemia.

In July of 2015, roughly 15 months after remission, he relapsed with open, weeping sores on his back. Biopsy demonstrated positivity for CD2, CD3, CD5, CD7, CD25 and CD30 in rare malignant T-cells. He was restarted on another 12-week course of alemtuzumab with little improvement in skin disease. In December, he was started on anti-CD30 treatment with $\mathrm{BV}$ due to the rare $\mathrm{CD} 30$ positive cells found in the excisional skin biopsy. He demonstrated a partial remission of cutaneous lymphoma following three treatments of BV
Correspondence: Jordan Senchak, Drexel University College of Medicine, Philadelphia, 2510 Maryland Road, Willowood Building Suite 175, Willow Grove, PA 19090, USA.

Tel: +1.724.714.2337. E-mail: js3759@drexel.edu

Key words: T-prolymphocytic leukemia; brentuximab vedotin; alemtuzumab.

Contributions: JS, collected data and authored the paper. PP, reviewed and edited the paper

Conflict of interest: the authors declare no potential conflict of interest.

Received for publication: 10 May 2016. Accepted for publication: 30 August 2016

This work is licensed under a Creative Commons Attribution-NonCommercial 4.0 International License (CC BY-NC 4.0).

(C) Copyright J. Senchak and P. Pickens, 2016

Licensee PAGEPress, Italy

Hematology Reports 2016; 8:6593

doi:10.4081/hr.2016.6593

that persisted for four months (Figures 1 and 2 ). When seen in February 2016 with progression of severe cutaneous involvement he commenced treatment with Pralatrexate to which he has currently demonstrated partial response of skin disease. Throughout his course, he has never developed progression to avert multiple myeloma.

\section{Discussion}

We report a case of a patient with T-PL with rare CD30 expression that demonstrated a partial response to treatment with a monoclonal antibody directed against CD30 in malignant lymphocytes. The skin lesions initially responded to treatment with CD52 specific alemtuzumab, as this is the first-line treatment for T-PL. Such treatment associates with an $80 \%$ remission rate, although remission tends to be brief. ${ }^{6-7} \mathrm{BV}$ is most often used in lymphoid malignancies that more characteristically express CD30, such as Hodgkin lymphoma and systemic anaplastic large cell lymphoma. ${ }^{8}$ The most common side effect is peripheral neuropathy and nausea/vomiting. ${ }^{9}$ In our patient, no neuropathy was noted and treatment was well tolerated. Our case shows that CD30 targeted therapy with BV has activity in recurrent, alemtuzumab resistant T-PL. Physicians caring for patients with T-PL should consider immunostaining these malignant $\mathrm{T}$ cells for CD30 expression and consider BV salvage treatment if cells express positivity. 


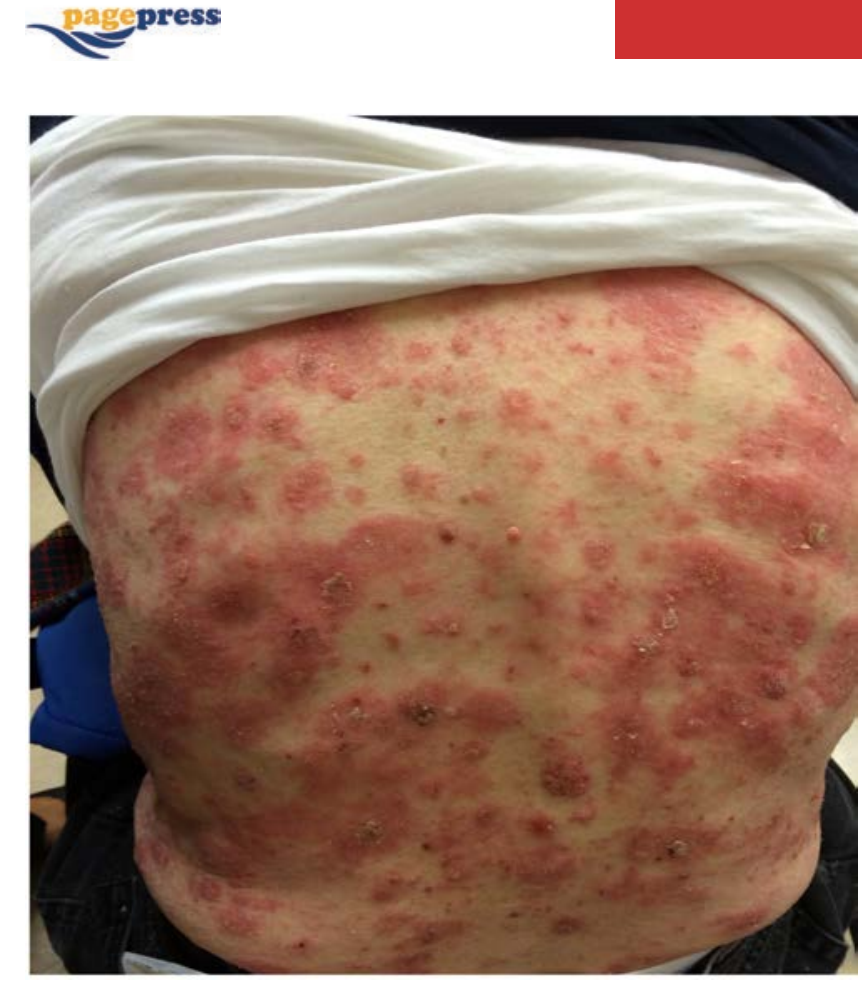

Figure 1. Pre brentuximab vedotin treatment, December 2015.

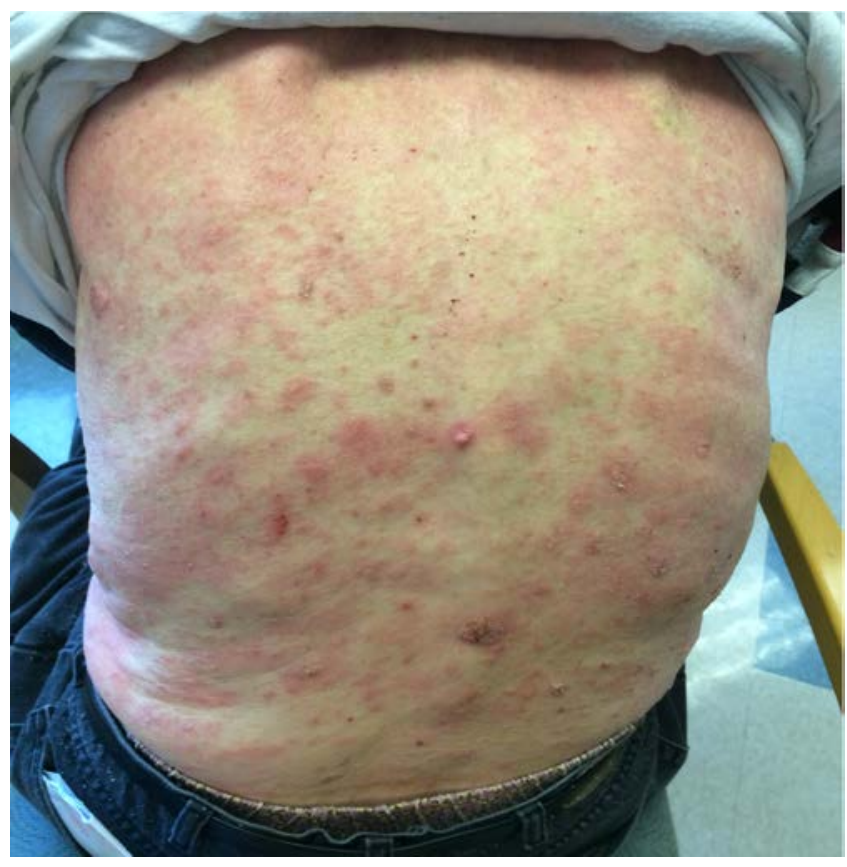

Figure 2. Post brentuximab vedotin treatment, February 2015.

\section{References}

1. Robak T, Robak P. Current treatment options in prolymphocytic leukemia. Med Sci Monit 2007;13:69-80.

2. Dearden C. Management of prolymphocytic leukemia. Hematology Am Soc Hematol Educ Program 2015;2015:361-7.

3. Hsi AC, Robird DH, Luo J, et al. T-cell prolymphocytic leukemia frequently shows cutaneous involvement and is associated with gains of MYC, loss of ATM, and TCL1A rearrangement. Am J Surg Path
2014;38:1468-83.

4. Dearden CE, Khot A, Else M, et al. Alemtuzumab therapy in T-cell prolymphocytic leukemia: comparing efficacy in a series treated intravenously and a study piloting the subcutaneous route. Blood 2011;118:5799-802.

5. Hasanali Z, Bikramajit S, Stuart A, et al. Epigenetic therapy overcomes treatment resistance in $\mathrm{T}$ cell prolymphpocytic leukemia. Sci Trans Med 2015;7:192.

6. Pawson R, Dyer MJ, Barge R, et al. Treatment of T-cell prolymphocytic leukemia with human CD52 antibody. J
Clin Oncol 1997; 15:2667-72.

7. Dearden C. How I treat prolymphocytic leukemia. Blood 2012;120:538-51.

8. Garnock-Jones, K. Brentuximab vedotin: a review of its use in patients with hodgkin lymphoma and systemic anaplastic large cell lymphoma following previous treatment failure. Drugs 2013;73:371-81.

9. Duvic M, Tetzlaff MT, Gangar P, et al. Results of a phase II trial of brentuximab vedotin for CD30+ cutaneous T-cell lymphoma and lymphomatoid papulosis. J Clin Oncol 2015;33:3759-65. 